\title{
Characterization of hydrogen bonding formation and breaking in semiconducting polymers under mechanical strain.
}

Andrea Gasperini ${ }^{1+}$, Ging-Ji Nathan Wang ${ }^{1}$, Francisco Molina-Lopez ${ }^{1 *}$. Hung-Chin Wu ${ }^{1}$, Jeffrey Lopez ${ }^{1}$, Jie Xu ${ }^{1}$, Shaochuan Lou², Dongshan Zhou², Gi Xue ${ }^{2}$, Jeffery B.-H. Tok and Zhenan $\mathrm{Bao}^{1 *}$

${ }^{1}$ Department of Chemical Engineering, Stanford University, Stanford, CA 94305-5025, USA

${ }^{2}$ Department of Polymer Science and Engineering, School of Chemistry and Chemical Engineering, Nanjing University, Nanjing 210093, China

${ }^{\ddagger}$ A.G, Brighlands Materials Center, Urmonderbaan 22, Geleen, The Netherlands, e-mail: andrea.gasperini@brightlandsmc.com

F. M-L, Apple One Apple Park way, MS 922-4HWE Cupertino, California, USA, e-mail: fmolinalopez@apple.com

*Corresponding author: Prof. Zhenan Bao, Department of Chemical Engineering, Stanford University, Stanford, CA 94305-5025, USA, e-mail: zbao@stanford.edu

\begin{abstract}
Diketopyrrolopyrrole (DPP) based donor-acceptor conjugated polymers, with increasing amount of weak H-bonding units, namely 2,6-pyridine dicarboxamide (PDCA), inserted as end groups in alkyl sidechains were prepared and investigated. In contrast to previously reported DPP polymers containing PDCA units as conjugation breakers along the polymer backbone, PDCA in the alkyl sidechains readily produced almost quantitative formation of intermolecular H-bonding even at low PDCA unit content $(<10 \mathrm{~mol} \%)$ as shown
\end{abstract}


by Fourier-transform infrared spectroscopy (FTIR). The efficient intermolecular H-bonding was further supported by the appearance of a pronounced vibronic shoulder in the UV-Vis spectra and a reduction of interlamellar spacing (from $24.02 \AA$ to $22.87 \AA$ ) compared to the neat DPP polymer. Increasing mol\% of PDCA units in sidechains of DPP conjugated polymers also has a clear effect on the thermal and mechanical properties of the films as investigated by Dynamic Mechanical Analysis (DMA). Polymers with a high loading of PDCA showed a linear increase in both tan delta intensity and temperature at which softening of film crosslinking occurs. In particular, at a comparable mol\%, polymers with PDCA units along the conjugated backbone showed a lower transition intensity and on average a $10{ }^{\circ} \mathrm{C}$ to $20{ }^{\circ} \mathrm{C}$ higher temperature required for H-bonding breaking. FTIR coupled with crack onset measurements showed that H-bonding breaking during tensile deformation happens only at strains close to crack onset. All these observations suggest that molecular engineering of conjugated polymers bearing H-bonding units has a strong influence on microstructure, thermal and mechanical properties of solution processed films and final energy dissipation mechanisms in stretchable electronics applications as well.

\section{Introduction}

The use of dynamic covalent crosslinking between elastomer chains is becoming a common method to provide higher toughness and self-healing properties to polymers. ${ }^{1-4}$ Inspired by nature, sacrificial bonds have been successfully incorporated into hydrogels ${ }^{5-9}$ and elastomers $^{10-12}$ to achieve improved strength and toughness. Among dynamic molecular bonds, there are covalent bonds such as Diels-alder cyclo-adducts and disulfide exchange; and supramolecular interactions, which include hydrogen bonding, $\pi-\pi$ stacking and metal-ligand coordination. ${ }^{13}$ In a dual network architecture, where the elastomer is held together by both a covalent network and hydrogen bonding, the hydrogen bonds act as sacrificial units by 
reversibly rupturing and re-forming to dissipate energy during stretching. This helps to render high stretchability and contributes to the increased fracture energy. ${ }^{14}$ The preferential rupture and dynamic features of the sacrificial hydrogen bonds "smooth out" the stress distribution and prevent local concentrations of stress, which facilitates chain sliding and orientation when force is applied, thus improving the overall strength of the rubber.

In organic electronics, ${ }^{15}$ the use of hydrogen bonding units has gained particular attraction owing to their spontaneous formation and healing ability when used in dielectric and semiconducting components. ${ }^{16}$ Indeed, Verduzco et al. ${ }^{17}$ showed that 2-ureido- $4[1 \mathrm{H}]-$ pyrimidone (UPy) end-groups in semiconducting polythiophene blends can prevent micrometer-scale phase separation in polymer blends by intermolecular hydrogen-bonding which consequently reduces polymer chain distance for solid state energy and/or electron transfer. Watkins and al. ${ }^{18}$ found instead that the stronger interactions between $\mathrm{COOH}$ functionalized bis-PCBA fullerenes and the oligo(ethylene oxide) side chains produced nanostructured donor/acceptor (D/A) interpenetrated networks which in turn led to improved photovoltaic performance as a result of enhanced morphological control of bulk heterojunction solar cells. Indeed, similarly to backbone engineering, side chain modifications have been shown to strongly influence solubility, molecular interactions, crystalline structure and film morphology in conjugated polymers. ${ }^{19}$ Intrachain charge transport is not affected by the introduction of H-bonding units in alkyl chains as encountered instead when functional conjugation breaker units are incorporated along the conjugated backbone. Moreover, the low glass transition temperature of alkyl chains $\left(\mathrm{T}_{\mathrm{g}}<-30{ }^{\circ} \mathrm{C}\right.$ by DMA) would ensure a sufficient degree of freedom even at room temperature for H-bonding units to interact in polymer films, compared to the more restricted backbone analogs $\left(\mathrm{T}_{\mathrm{g}}>120^{\circ} \mathrm{C}\right.$ by DMA). Yao et al. reported DPP-quaterthiophene conjugated polymers with high charge carrier mobility, in which part of the branching alkyl chains are replaced by urea-containing alkyl chains. ${ }^{20}$ Side chain 
engineering via incorporation of hydrogen-bonding was shown here to significantly affect inter-chain interactions leading to stronger self-aggregation during thin film formation. The increase in both domain size and order improved the charge carrier mobilities and photovoltaic performance for conjugated polymers with $10 \mathrm{wt} \%$ of urea groups along alkyl sidechains. ${ }^{20}$ In skin-inspired electronics, non-covalent cross-linking was reported by our group as a design concept to produce stretchable semiconductors which can help dissipate mechanical energy involved during tensile deformation. In the initial design motif, a 2,6-pyridine dicarboxamide (PDCA) unit with weak hydrogen bonding strength was incorporated along the rigid polymer backbone of a DPP-based polymer. Owing to their non-conjugated nature, the PDCA units also act as main chain conjugation breaker which produced a significant reduction of film Young's modulus and an increase in crack onset strain compared to the pristine DPP polymer. ${ }^{21} \mathrm{We}$ further reported the use of solution processable isoindigo polymers incorporating the same PDCA units along the backbone and in the alkyl side chains as a binders for sulfur electrodes. ${ }^{22}$ Depending on the H-bonding unit position, different sulfur binding properties were observed. PDCA pyridine groups along the polymer backbone provided high affinity to polysulfide species leading to effective coating on various carbonaceous substrates to provide highly stable sulfur electrodes. However, polymers with PDCA in the alkyl chains presented only one active H-bonding sites compared to the two amide groups for PDCA along the backbone and significant different solubilities in organic solvents. Therefore, no clear structure-properties relationships can be concluded when comparing polymers with PDCA units in different positions.

Although the incorporation of PDCA in alkyl chain has been indirectly proposed as a promising design motif to enhance supramolecular interactions at the molecular level between hydrogen bonding units, little is known about both the degree of $\mathrm{H}$-bonding formation and the parameters that regulates their dynamic nature when incorporated in semi-crystalline conjugated polymers 
versus highly stretchable elastomers or hydrogels. Furthermore, incorporation of H-bonding units in alkyl chains may be used as a design motif to maintain high charge carrier mobilities while adding new functionalities in conjugated polymers. Here, we systematically investigated in detail the influence that different position of PDCA in DPP polymers (sidechain vs. mainchain) have on the amount of H-bonding formed and the resulting influence on the degree of crystallinity. Combination of thermal and spectroscopic methods provided insights into the thermal stability of such systems and the formation and breaking of H-bonding units in the solid state. Finally, we developed guidelines for molecular design of these weakly cross-linked systems with different levels of cross-linking density and elucidated energy dissipation mechanisms during mechanical strain.

\section{Results and Discussions}

Synthesis and H-bonding characterization. The DPP-TVT conjugated polymers synthesized in this work with different mol\% of PDCA groups on the side chains and along the conjugated backbone is presented in Figure 1. The detailed preparation procedures for the monomers are included in Supporting Information (Figure S1 and Figure S2). By varying the molar ratio between 1 and 9 from 1:0, 0.95:0.05 to 0.85:0.15 and 0.7:0.3, the regio-random polymers with PDCA units randomly incorporated were synthetized through Stille coupling to obtain DPPTVT-0, DPP-TVT-10, DPP-TVT-30 and DPP-TVT-60, respectively. Each polymer was subjected to Soxhlet extraction with methanol, hexane, and acetone to remove the remaining monomers and oligomers. For comparison, a non-interacting PDCA derivative polymer, where amide groups are substituted with ester groups, was also prepared for comparison in order to decouple PDCA steric effect vs. H-bonding effect.. All polymers synthetized can be dissolved in chloroform $\left(\mathrm{CHCl}_{3}\right)$, 1,1,2,2-tetrachloroethane, toluene, and other aromatic 
solvents. Moreover, the in ${ }_{n} u e n c e$ of chain entanglements was minimized by synthesizing polymers with molecular weights below the entanglement molecular weight which for semiconducting polymers such as regioregular poly(3-hexylthiophene) (P3HT) is around 20 $\mathrm{kDa}^{23}$ The synthesized conjugated polymers had number-averaged molecular weights in the range of $17 \mathrm{kDa}<\mathrm{M}_{\mathrm{n}}<20 \mathrm{kDa}$ and the amount of H-bonding PDCA units are confirmed by ${ }^{1} \mathrm{H}-\mathrm{NMR}$, in molar percentage ranging from $10 \mathrm{~mol} \%$ to $60 \mathrm{~mol} \%$ as reported in Table 1 . The chemical structures of DPP-TVT-0, DPP-TVT-10, DPP-TVT-30, DPP-TVT-60 and as well as DPP-TVT-60E were verified by ${ }^{1} \mathrm{H}-\mathrm{NMR}$ and reported in Supporting information.
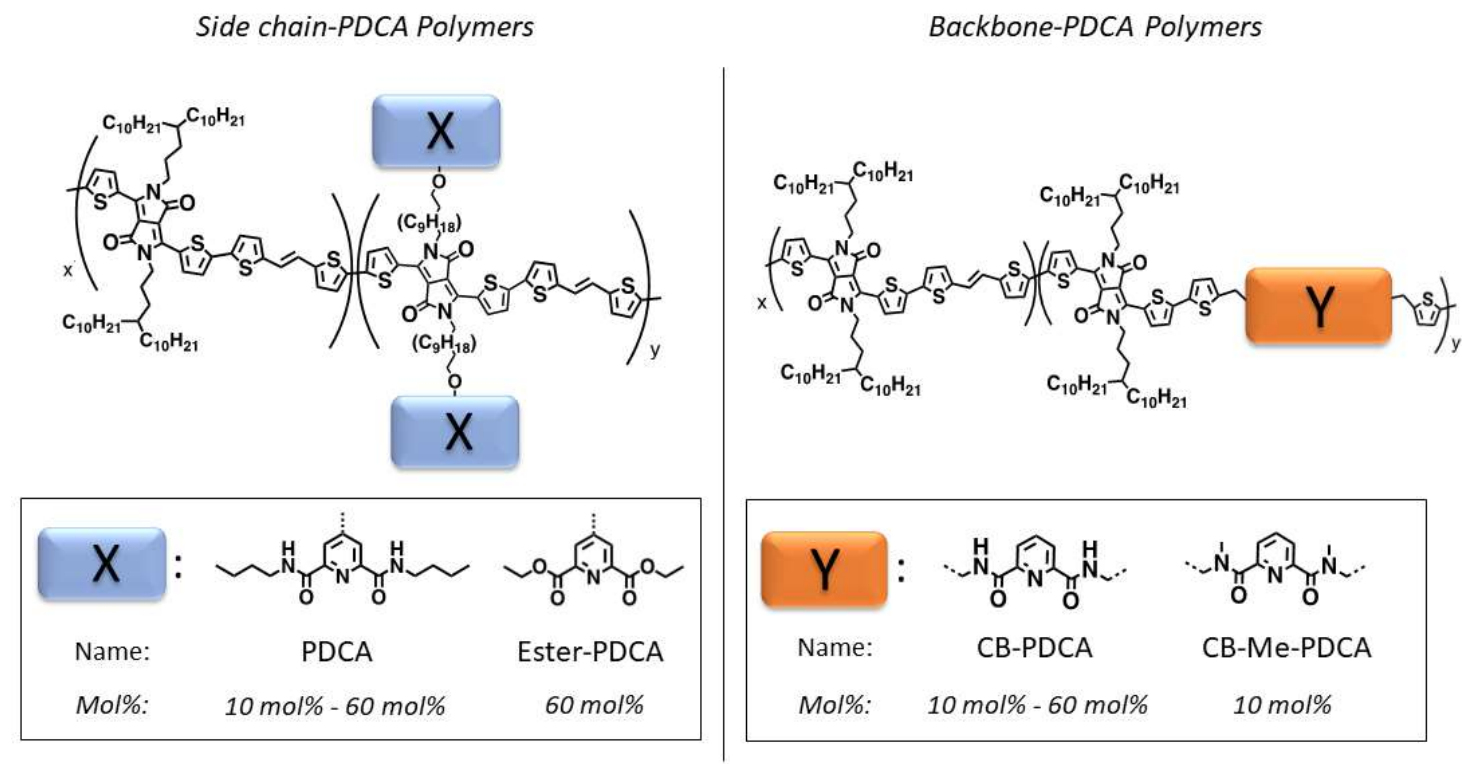

Figure 1. Schematic showing the chemical structure of the DPP-based polymers used in this study and their respective H-bonding groups PDCA units. 
Table 1. Molecular Weight and Physical Properties of the different H-bonding DPP Polymers synthetized

\begin{tabular}{|c|c|c|c|c|c|c|c|c|c|}
\hline Polymer & $x: y$ & $\begin{array}{l}M_{n} \\
\left(\mathrm{~kg} \mathrm{~mol}^{-1}\right)^{a}\end{array}$ & $\begin{array}{l}M_{w} \\
\left(\mathrm{~kg} \mathrm{~mol}^{-1}\right)^{b}\end{array}$ & $P D I^{c}$ & $D P_{n}{ }^{d}$ & $\begin{array}{l}T_{m 1} \\
\left({ }^{\circ} \mathrm{C}\right)^{e}\end{array}$ & $\begin{array}{l}\Delta H_{1} \\
\left(J g^{-1}\right)^{f}\end{array}$ & $\begin{array}{l}T_{m 2} \\
\left({ }^{\circ} C\right)^{g}\end{array}$ & $\begin{array}{l}\Delta H_{2} \\
\left(J g^{-1}\right)^{h}\end{array}$ \\
\hline DPP-TVT-0 & 100:0 & 20.2 & 42.4 & 2.1 & 10.2 & 135.7 & 0.19 & 313.1 & 2.5 \\
\hline$D P P-T V T-30$ & $70: 30$ & 19.3 & 42.4 & 2.1 & 9.3 & 142.0 & 0.10 & 330.3 & 6.1 \\
\hline DPP-TVT-60 & $40: 60$ & 17.5 & 42.0 & 2.4 & 8.5 & 112.7 & 0.11 & 339.0 & 10.5 \\
\hline DPP-TVT-60E & $40: 60$ & 19.1 & 42.1 & 2.1 & 9.2 & 137.3 & 0.12 & - & - \\
\hline$D P P-T V T-10 C B$ & $90: 10$ & 20.0 & 40.0 & 2.0 & 10.1 & - & - & 365.2 & 7.1 \\
\hline DPP-TVT-30CB & $70: 30$ & 19.2 & 42.0 & 2.1 & 9.9 & - & - & 320.3 & 1.7 \\
\hline$D P P-T V T-60-C B$ & $40: 60$ & 10.1 & 25.2 & 2.4 & 5.1 & - & - & - & - \\
\hline$D P P-T V T-10-C B-M e$ & $90: 10$ & 21.0 & 43.4 & 2.2 & 10.5 & - & - & 361.2 & 6.1 \\
\hline
\end{tabular}

${ }^{a}$ Number-average molecular weight. ${ }^{b}$ Weight-average molecular weight. ${ }^{C}$ Weight dispersity, defined as Mw/Mn. ${ }^{d}$ Degree of polymerization. ${ }^{e}$ Side-chain melting temperature measured by DSC. ${ }^{F}$ Enthalpy of fusion of side chain measured by DSC, ${ }^{g}$ Backbone melting temperature measured by DSC, ${ }^{h}$ Enthalpy of fusion of backbone measured by DSC

In order to confirm the formation of inter-chain hydrogen-bonding, ${ }^{1} \mathrm{H}$ NMR spectra of DPPTVT-60 (60 mol\% of PDCA) were measured at different temperatures in 1,1,2,2tetrachloroethane- $\mathrm{d}_{2}(20.0 \mathrm{mg} / \mathrm{mL})$. The ${ }^{1} \mathrm{H}$ NMR signal of the amide $\mathrm{N}-\mathrm{H}$ proton was detected at $8.94 \mathrm{ppm}$ at $333 \mathrm{~K}$. The ${ }^{1} \mathrm{H}$ NMR signal gradually upfield-shifted to $8.91 \mathrm{ppm}$ at $353 \mathrm{~K}, 8.88$ ppm at $373 \mathrm{~K}$, and $8.85 \mathrm{ppm}$ at $393 \mathrm{~K}$ (Figure S13). Moreover, addition of a protic solvent such as deuterated methanol resulted in a disappearance of the $\mathrm{N}-\mathrm{H}$ amide proton due to proton exchange. Such an observation further confirms the formation of inter-chain hydrogen-bonding due to amide groups incorporated in the sidechains. In H-bonding polymers, also the effect of 
concentration on the $\mathrm{N}-\mathrm{H}$ position was studied in the range from 1 to $80 \mathrm{mg} / \mathrm{ml}$ in deuterated TCE. No concentration-dependent shift was observed in the range of concentrations investigated. This result seems to suggest that already at concentrations as low as $1 \mathrm{mg} / \mathrm{ml}$ the limiting chemical shift for the fully hydrogen bonded state was attained.

For an amide proton equilibrating between a non hydrogen-bonded state and a hydrogenbonded state, the equilibrium constant $\mathrm{K}_{\mathrm{eq}}(\mathrm{T})$ between the two states may be extracted from chemical shift $(\partial)$ of $\mathrm{N}-\mathrm{H}$ if the limiting chemical shifts for the non-hydrogen-bonded and hydrogen-bonded states are known: ${ }^{24}$

$$
\mathrm{K}_{\mathrm{eq}}(\mathrm{T})=\left(\partial_{\mathrm{obs}}-\partial_{\mathrm{n}}\right) /\left(\partial_{\mathrm{b}}-\partial_{\mathrm{obs}}\right)
$$

where $\partial_{\mathrm{obs}}$ is the observed chemical shift, $\partial_{\mathrm{n}}$, is the limiting chemical shift for the nonhydrogen-bonded state, and $\partial_{\mathrm{b}}$, is the limiting chemical shift for the fully hydrogen bonded state. Determining the equilibrium constant at several temperatures allows one to evaluate the $\Delta \mathrm{H}$ and $\Delta \mathrm{S}^{\circ}$ for the two-state process, via van't Hoff analysis ${ }^{25}$ :

$$
\ln \mathrm{K}_{\mathrm{eq}}=(-\Delta \mathrm{H} / \mathrm{R})(1 / \mathrm{T})+\left(\Delta \mathrm{S}^{\circ} / \mathrm{R}\right)
$$

The van't Hoff analysis for a simple PDCA unit (no para-alkoxy group) at different temperatures in 1,1,2,2- tetrachloroethane- $\mathrm{d}_{2}$ over the temperature range $293-393 \mathrm{~K}$ (10 K step) and at concentrations ranging from $2.5 \mathrm{M}$ to $0.002 \mathrm{M}$ was performed (Figure S14). ${ }^{21}$ The solution dimerization energies of intermolecular PDCA H-bonding $(\Delta \mathrm{H})$ was found to be 21.6 $\pm 2.9 \mathrm{~kJ} / \mathrm{mol}$ using Equation 2. In comparison, the "strong" ureidopyrimidinone (UPy) dimer units with four H-bonding sites has a dimerization energy of $70 \mathrm{~kJ} / \mathrm{mol}{ }^{26}$ while the weak aminopyridine H-bonding group has a reported dimerization energy of $25 \mathrm{~kJ} / \mathrm{mol}^{24}$. Thus, 
within this range H-bonding strength, PDCA units can be readily considered as a "weak" hydrogen-bonding group. In the final polymer structure, PDCA in the side-chain has an electron-donating group at the para position. Substituents onto H-bonding forming aromatic units are known to alter both steric interactions and the acidity of the interacting groups i.e. amides and acids. However, the weak acidity of amides groups and the dominant steric hindrance of the conjugated backbone itself significantly reduce the effect that the para-alkoxy group can have on hydrogen bonding formation in solution and solid state.

UV-vis spectroscopy. Despite the low H-bonding energy, PDCA units strongly influence thin film absorption spectra as shown in Figure 2. The $\pi$ - $\pi^{*}$ transition was recorded around 450 $\mathrm{nm}$ for all polymers measured in the solid state. The charge transfer peak between 600 and 900 $\mathrm{nm}$ showed two clear vibronic bands: the one at higher energy $(0-1)$ and one at lower energy $(0-0)$ which is normally attributed to polymer aggregation. ${ }^{21}$ When all spectra are normalized to the $0-1$ vibronic peak, the absorption peak (0-0) at around $810 \mathrm{~nm}$ becomes more pronounced for thin films of polymers bearing H-bonding units. Interestingly, incorporation of H-bonding PDCA units promoted strong inter-chain interactions even in such stiff systems like DPP-TVT conjugated polymers. The ester bearing polymer (DPP-TVT-60E), on the other hand, showed the weakest absorption at $810 \mathrm{~nm}$. This indicates that the presence of bulky but not interacting PDCA-like units decreased the degree of aggregation of polymer chains in thin films. Therefore, despite of being bulky, PDCA units can improve aggregation and intermolecular interaction by means of H-bonding formation. 


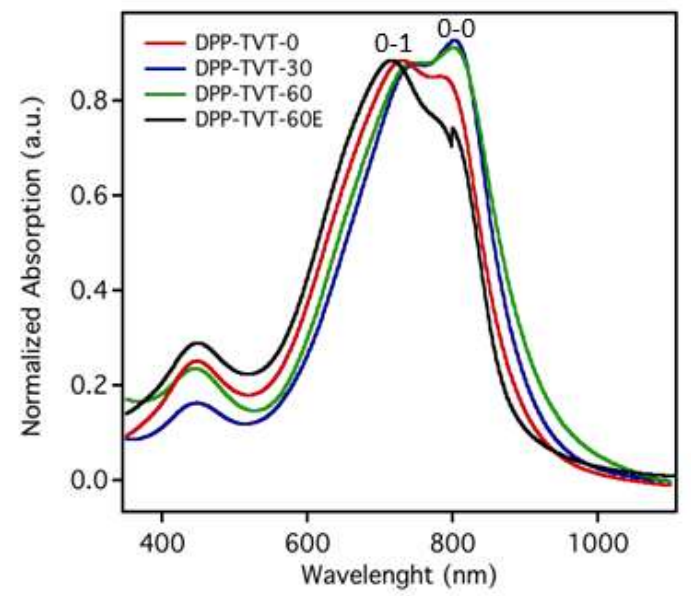

Figure 2. Normalized UV-vis absorption spectra of the different DPP polymers in spin-coated thin film on glass

Optical microscopy and dichroic ratio. To gain further insights into the molecular level changes during stretching, the degree of polymer chain alignment under strain was measured using polarized ultraviolet-visible spectroscopy and quantified using dichroic ratios. As previously reported for standard DPP-TVT-0 polymers ${ }^{27}$, the dichroic ratio initially increased to 1.2 due to a strain-induced chain alignment. Above $30 \%$ strain, however, the dichroic ratio remained constant (Figure S30); this was caused by chain relaxation mechanism due to crack formation upon increasing the strain from $30 \%$ to $100 \%$ above the crack onset $(\mathrm{CoS})$. This observation indicates that fully conjugated DPP-TVT-0 polymers is not ductile and, indeed, we observed that cracks began to form even at low strains $(<30 \%)$ (Figure S25). Previously, Oh et al. ${ }^{27}$ instead reported dichroic ratios for polymers bearing PDCA units as conjugation breakers along the polymer backbones which increased linearly to 1.8 , as strain increased from $30 \%$ to $100 \%$. $^{21}$ This result was supported by the observation of crack onset above $100 \%$ and a retention of charge carrier mobility during tensile stretching. In contrast, polymers bearing the PDCA units in the alkyl chains showed a slight increase in the DR until $30 \%$, suggesting 
polymer chain alignment, followed by a flattening of the DR in conjunction with the observed microscopic cracks above $30 \%$ strain, similar to the neat DPP-TVT-0 polymer.

Charge carrier mobility. To probe the effect of hydrogen-bonding sites located in the side chains on the charge transport property of solution processed thin films, bottom-gate/ topcontact (BGTC) field-effect transistors (FETs) were fabricated following standard procedures (Supporting Information) ${ }^{29}$ and the devices were measured in air. Figure S23 shows the respective transfer and output characteristics of FETs with thin films of these conjugated polymers after thermal annealing at $150^{\circ} \mathrm{C}$ for 30 minutes in nitrogen. They all exhibited typical p-type semiconducting behavior. The hole mobilities $\left(\mu_{\mathrm{h}}\right)$ were extracted by fitting the linear part of the plot of $\left|\mathrm{I}_{\mathrm{DS}}\right|^{1 / 2} \mathrm{vs} \mathrm{V}_{\mathrm{GS}}$. Addition of increasing amount of PDCA units in the alkyl chains up to $60 \mathrm{~mol} \%$ caused a drop of about $50 \%$ in charge carrier mobility from $1.03 \pm$ 0.10 to $0.62 \pm 0.21 \mathrm{~cm}^{2} / \mathrm{Vs}$. In comparison, devices fabricated with $20 \mathrm{wt} \%$ of PDCA as conjugation breaker, were reported by $\mathrm{Oh}$ et al. with mobilities already below 0.58 $\mathrm{cm}^{2} / \mathrm{Vs}$. Increasing mol\% of PDCA units also induced a slight decrease in on/off ratio. This result confirmed our hypothesis that having the H-bonding sites in sidechain rather than conjugation breakers produce a more moderate reduction in charge carrier mobility. Therefore, incorporation of H-bonding units in alkyl chains may be used as a design motif to maintain high charge carrier mobilities while adding new functionalities in conjugated polymers.

Table 2. Device characterization parameters for device fabricated from DPP-TVT polymers with PDCA units in alkyl chains.

\begin{tabular}{|l|l|l|l|l|l|}
\hline \multicolumn{1}{|c|}{ Polymer } & \multicolumn{1}{|c|}{ Rpm } & substrate & $\begin{array}{c}\boldsymbol{\mu}_{\mathbf{h}} \\
{\left[\mathbf{c m}^{2} \mathbf{V}^{-1} \mathbf{s}^{-1}\right]}\end{array}$ & \multicolumn{1}{|c|}{$\mathbf{I}_{\text {on }} / \mathbf{I}_{\text {off }}$} & $\mathbf{V}_{\mathbf{t}}(\mathbf{V})$ \\
\hline DPP-TVT-0 & 1500 & OMTS & $1.03 \pm 0.10$ & $>6^{*} 10^{5}$ & 3,3 \\
\hline DPP-TVT-10 & 1500 & OMTS & $0.71 \pm 0.14$ & $>5^{*} 10^{5}$ & 2.5 \\
\hline DPP-TVT-30 & 1500 & OMTS & $0.62 \pm 0.23$ & $>4 * 10^{5}$ & 3.1 \\
\hline DPP-TVT-60 & 1500 & OMTS & $0.60 \pm 0.21$ & $>1 * 10^{4}$ & 3.3 \\
\hline
\end{tabular}


Grazing Incidence X-ray Diffraction (GIXD). Thin films of DPP-TVT with PDCA units in the alkyl chains were spin coated onto OTS treated silicon substrates and measured by GIXD in the as-cast state. Both series of samples showed clear out-of-plane (h00) diffraction pattern suggesting a preferential edge-on orientation of crystalline lamellae as shown in Figure 3c. Experimental d-spacing values and FWHM are reported in Table 2. The presence of alkyl chains with PDCA units slightly reduced the lamellae d-spacing from $24.02 \AA$ for the reference polymer to $23.50 \AA$ and $22.87 \AA$ for polymers with $30 \mathrm{~mol} \%$ and $60 \mathrm{~mol} \%$ of PDCA, respectively. Interestingly, after thermal annealing at $150{ }^{\circ} \mathrm{C}$ DPP-TVT-0 and DPP-TVT-30 showed no variation in d-spacing, whereas polymers with higher loadings of PDCA showed an increase in d-spacing of $0.7 \AA$. The full width half maximum (FWHM) qualitatively gives information about the distribution of lamellae orientation. The lower the FWHM is an indication of a more oriented crystalline domains in the out-of-plane direction ${ }^{28}$. The reference sample appeared to be slightly more oriented than the polymers bearing PDCA units in the ascast form as indicated by the lower FWHM for DPP-TVT-0. This may be due to the weak Hbonding between side chains which restricted the polymer chains from having maximal $\pi-\pi$ interaction. After annealing all polymers appeared to have more oriented crystalline domains on average (smaller FWHM). However, thermal annealing, in general, seems to slightly increase lamellae spacing from the as cast state while reducing the influence of bonded PDCA units on the thin film microstructure. 

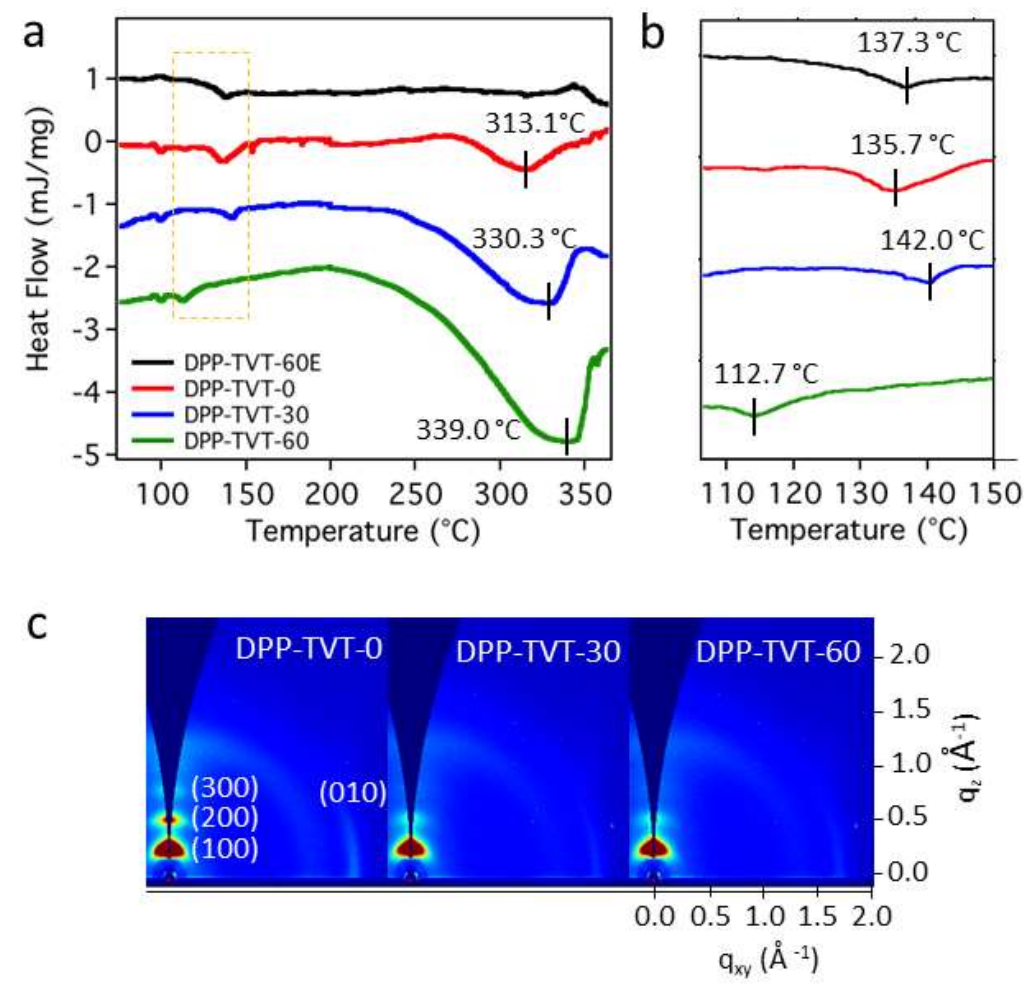

Figure 3. a) Differential scanning calorimetry ( $1^{\text {st }}$ heating curves, exotherm up) of polymers bearing PDCA units in the alkyl chains b) Inset into the side chain melting endotherms $c$ ) Grazing-incidence X-ray diffractograms of DPP polymers with different moll\% of PDCA units in the alkyl chains. Polymers were spin-coated from $5 \mathrm{mg} \mathrm{mL} \mathrm{m}^{-1}$ chlorobenzene solutions on Si substrates and measured as cast. All images were scaled for exposure time and illuminated volume for qualitative comparison across samples.

Table 3. Crystallographic parameters of side-chain PDCA films in the as-cast state

\begin{tabular}{c|c|c|c|}
\hline Polymer & $\mathbf{q}_{\mathbf{z}}(\mathbf{2 0 0})^{\mathbf{a}}$ & $\begin{array}{c}\text { Lamellar Spacing } \\
(\AA)^{\mathbf{a}}\end{array}$ & $\begin{array}{c}\text { Lamellar peak } \\
\text { FWHM }\left(\AA^{-1}\right)^{\mathbf{a}}\end{array}$ \\
\hline DPP-TVT-0 & 0.5230 & 24.02 & 0.0800 \\
DPP-TVT-30 & 0.5346 & 23.50 & 0.0972 \\
DPP-TVT-60 & 0.5350 & 23.48 & 0.0989 \\
\hline
\end{tabular}

${ }^{a}$ Extracted from fitting the (200) diffraction peak 
Thermal properties. On the basis of thermogravimetric analysis (TGA) data shown in Figure S20, the decomposition temperatures (measured at 5\% weight loss) of the DPP-TVT-0, DPPTVT-60 are all higher than $400{ }^{\circ} \mathrm{C}$. The effect of incorporation of H-bonding groups into alkyl chains and DPP backbone on the thermal properties of this class of conjugated polymers was investigated by means of differential scanning calorimetry (DSC) to record melting temperatures and enthalpies (first order transitions) and the more sensitive AC-Chip DSC to accurately measure backbone glass transitions temperatures (second order transitions). ${ }^{30} \mathrm{~A}$ differential AC - chip calorimeter is indeed capable of measuring minimal changes in heat capacity at the glass transition in nanometer - thin films in a broad range of temperatures. From DSC endotherms of Figure 3a, the reference polymer DPP-TVT-0 showed an expected sidechain melting temperature $\left(\mathrm{T}_{\mathrm{m} 1}\right)$ usually reported at $135.7^{\circ} \mathrm{C}$. Despite a slight increase in $\mathrm{T}_{\mathrm{m} 1}$ for DPP-TVT-30 up to $142{ }^{\circ} \mathrm{C}$, DPP-TVT-60 has a pronounced reduction in $\mathrm{T}_{\mathrm{m} 1}$ down to $112.79^{\circ} \mathrm{C}$. These results suggest that the PDCA units affect the crystallization of the side chains, likely due to increased irregularity in spacing between the repeating side chain locations with introduction of more PDCA units. A further prove of the incorporation of PDCA in the side chain crystalline regions emerges from the thermogram of the analog DPP-TVT-60E, which despite the same amount of not interacting motif in the alkyl chains, showed a side chain crystals' melting of $137.30^{\circ} \mathrm{C}$ as the DPP-TVT-0 polymer. This observation suggests that the ester units in DPP-TVT-60E are not participating in the formation of crystalline domains, leaving the crystals formation driven by the interaction of branched alkyl chains exclusively. Unexpectedly, the melting temperature of the conjugated backbone instead increased gradually from $313{ }^{\circ} \mathrm{C}$ to $339{ }^{\circ} \mathrm{C}$ for DPP-TVT-0 and DPP-TVT-60, respectively (Figure 3a). The enthalpy of fusion for the backbone seems also to drastically increase at higher PDCA loadings. Again, this is consistent with the increased aggregation by UV-Vis. As reported by Yao et al. ${ }^{31}$, 
in which urea groups were inserted into alkyl side chains, the presence of H-bonding units was thought to have improved backbone packing by displaying a higher backbone melting temperature and an increase in the overall crystallinity of the film. The increase in backbone $\mathrm{T}_{\mathrm{m}}$ is a result of increased molecular interaction, which could be an indication of an increase in crystalline domain packing density or more perfection and longer range order. The strength of molecular interaction within the crystalline domain depends on chain structure: regularity (tacticity), chain stiffness, and interacting groups ( i.e. $\pi-\pi$ stacking or H-bonding). Therefore polymers with increased fraction of H-bonding sites which softly cross-link polymer chains inside the crystalline domains will need more thermal energy to melt, ${ }^{32}$ which may explain the observation above that the enthalpy of fusion $(\Delta \mathrm{H})$ was influenced by the presence of increasing amount of PDCA units. Indeed, an increase in $\Delta \mathrm{H}$ from $2.1 \mathrm{~J} /$ gr to $10.2 \mathrm{~J} / \mathrm{gr}$ was observed for DPP-TVT-0 and DPP-TVT-60 respectively. A backbone crystallization temperature for DPP-TVT-0 could be observed during cooling at $\mathrm{T}_{\mathrm{c} 2}=303{ }^{\circ} \mathrm{C}$ (Figure S21). However, the polymers with H-bonding PDCA units did not show crystallization upon cooling. The polymer with ester analog of PDCA instead showed no clear backbone melting in the range of temperatures measured (Figure 3a). The above results made us conclude that the incorporation of H-bonding units in the side chains led to participation of H-bonding in both side chains and backbone crystalline regions. To better understand the effect of PDCA units on polymer chains dynamics and free volume, the $\mathrm{T}_{\mathrm{g}}$ of $\mathrm{H}$-bonding polymers were measured accurately using AC-Chip DSC and dynamic mechanical analysis (DMA) as normal DSC is not sensitive enough. ${ }^{33}$ The $\mathrm{T}_{\mathrm{g}}$ values measured by DSC and DMA are summarized and compared in Table 3. Complete DSC and DMA curves for all polymers measured can be found in Supporting information (Figure S29). 

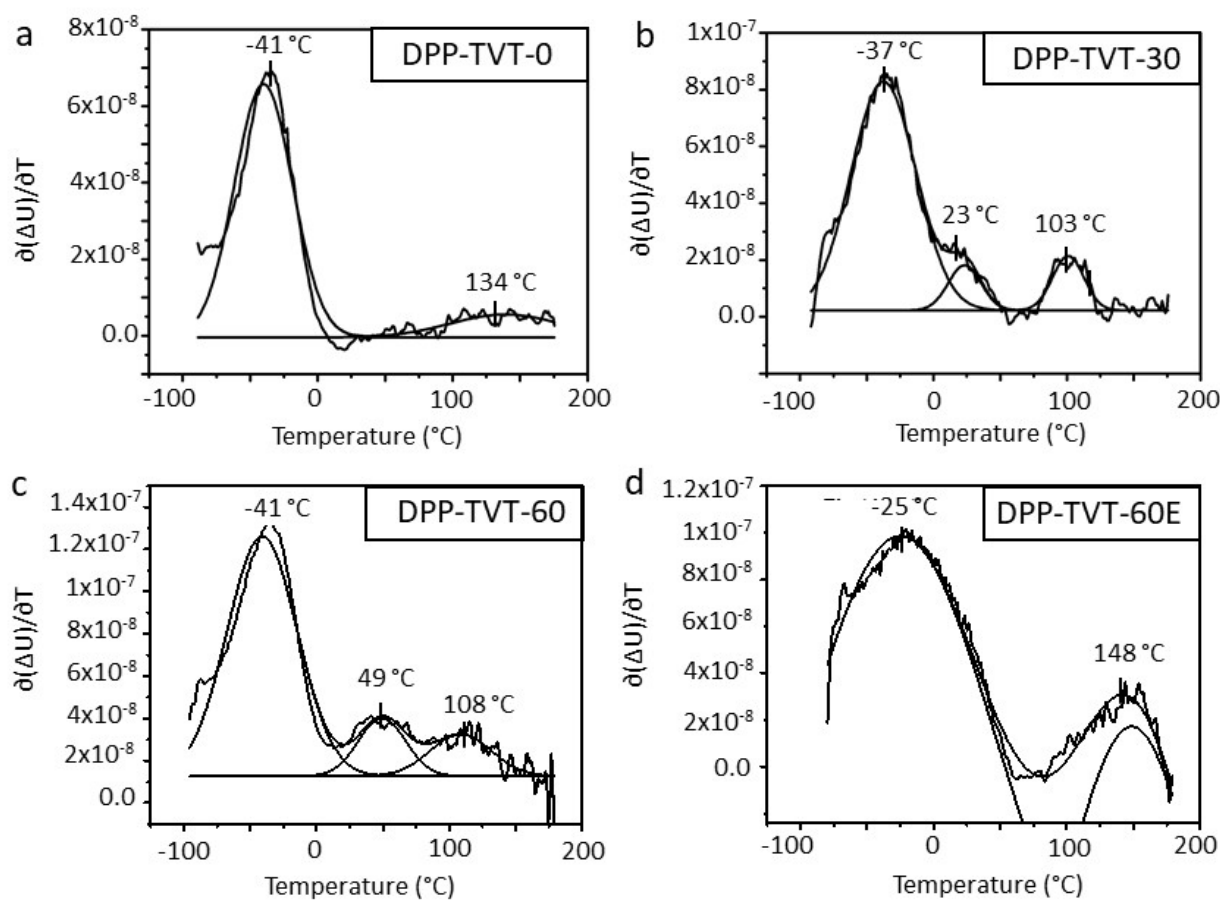

Figure 4. Baseline corrected first heating AC-CHIP DSC scans for semiconducting polymers bearing H-bonding units in the alkyl chains at A) DPP-TVT-0, B) DPP-TVT-30, C) DPP-TVT60 and D) DPP-TVT-60E.

In particular, AC-chip DSC was used to get insights on the effect that $\mathrm{H}$-bonded units have on chain arrangement in the amorphous regions in large temperature window compared to DMA through measuring the change in glass transition temperatures $\left(\mathrm{T}_{\mathrm{g}}\right)$ for both alkyl chains and backbone PDCA. Notably, from Figure $4 \mathrm{a}$ it is possible to recognize two characteristic transition of conjugated polymers. The $\alpha_{\mathrm{PE}}$ transition represents the softening of polyethylenelike chains, ${ }^{34}$ i.e. alkyl side chains $\left(\mathrm{T}_{\mathrm{g}}^{1}\right)$, and it is normally registered at a temperature below $-30{ }^{\circ} \mathrm{C}$. Instead backbone glass transition temperature $\left(\mathrm{T}_{\mathrm{g}}^{3}\right)$ are less pronounced and usually reported at temperatures ranging from $\mathrm{RT}$ to $140{ }^{\circ} \mathrm{C}$ for conjugated polymers. ${ }^{23}$ Backbone $\mathrm{T}_{\mathrm{g}}$ is strongly affected by the degree of polymerization, monomer chemical structure and regioregularity. Interestingly, the incorporation of H-bonding PDCA units has a drastic effect on DPP-TVT's $\mathrm{T}_{\mathrm{g}}$ which resulted in a clear decrease in backbone $\mathrm{T}_{\mathrm{g}}$ from $134^{\circ} \mathrm{C}$ to $96^{\circ} \mathrm{C}$ for 
DPP-TVT-0 vs. DPP-TVT-30 (Figure 4b). This may be due to increased disorder induced by H-bonding PDCA units. However, as PDCA fraction increased to DPP-TVT-60, the $\mathrm{T}_{\mathrm{g}}$ increased slightly up to $108^{\circ} \mathrm{C}$, which may be due to the contribution of soft crosslinking by PDCA units exceeding that from increased disorder. On the other hand, DPP-TVT-60E with ester version of the PDCA showed a backbone $T_{g}$ even higher than DPP-TVT-0. The above observations suggest that $\mathrm{H}$-bonding soft crosslinking also impacts the chain interactions in the amorphous regions. Unexpectedly, the AC-Chip DSC thermograms of Figure $4 \mathrm{~b}$ and 4c, showed an intermediate transition occurring between alkyl chain and backbone glass transition temperatures. Interestingly the intensity of such transition, coded $\left(\mathrm{T}_{\mathrm{g}}^{2}\right)$, seemed to be proportional to the amount of PDCA incorporated into the structure.

Table 4. Glass transitions temperatures for different DPP polymers measured by AC-chip DSC and DMA.

\begin{tabular}{|c|c|c|c|c|c|c|c|}
\hline \multirow[b]{2}{*}{ Polymer } & \multicolumn{3}{|c|}{$\mathrm{T}_{\mathrm{g}}^{1}$} & \multicolumn{3}{|c|}{$\mathrm{T}_{\mathrm{g}}^{2}$} & \multirow{2}{*}{$\begin{array}{c}\mathrm{T}_{\mathrm{g}}^{3} \\
\text { AC-DSC } \\
\left({ }^{\circ} \mathrm{C}\right)^{\mathrm{a}}\end{array}$} \\
\hline & $\begin{array}{l}\text { AC-DSC } \\
\left({ }^{\circ} \mathrm{C}\right)^{\mathrm{a}}\end{array}$ & $\begin{array}{l}\text { Tan } \delta \\
\left({ }^{\circ} \mathrm{C}\right)^{\mathrm{b}}\end{array}$ & $\begin{array}{l}\mathrm{E}^{\prime \prime} \\
\left({ }^{\circ} \mathrm{C}\right)^{\mathrm{c}}\end{array}$ & $\begin{array}{l}\text { AC-DSC } \\
\left({ }^{\circ} \mathrm{C}\right)^{\mathrm{a}}\end{array}$ & $\begin{array}{l}\text { Tan } \delta \\
\left({ }^{\circ} \mathrm{C}\right)^{b}\end{array}$ & $\begin{array}{l}\mathrm{E}^{\prime \prime} \\
\left({ }^{\circ} \mathrm{C}\right)^{\mathrm{c}}\end{array}$ & \\
\hline DPP-TVT-0 & -41 & -20 & -39 & - & & & 134 \\
\hline DPP-TVT-30 & -37 & -20 & -34 & 23 & 26 & 12 & 103 \\
\hline DPP-TVT-60 & -41 & -25 & -45 & 49 & 55 & 38 & 108 \\
\hline DPP-TVT-60E & -25 & 3 & -28 & - & - & - & 148 \\
\hline DPP-TVT-10CB & -35 & -27 & -40 & - & - & - & 113 \\
\hline DPP-TVT-30CB & -30 & -20 & -42 & - & 47 & 33 & 103 \\
\hline DPP-TVT-60-CB & -33 & -27 & -41 & - & 60 & 45 & 103 \\
\hline DPP-TVT-10-CB-Me & -35 & - & - & - & - & - & 113 \\
\hline
\end{tabular}


${ }^{a}$ Glass transition temperature measured by AC-chip DSC. ${ }^{b}$ Glass transition temperature defined by tan $\delta$ as measured by dynamic mechanical analysis. ${ }^{c}$ Glass transition temperature defined by the loss modulus ( $\left.E^{\prime \prime}\right)$ as measured by dynamic mechanical analysis.

Dynamic mechanical analysis was indeed used to investigate the effects of structural and processing parameters on glass transition temperatures $\left(\mathrm{T}_{\mathrm{g}}^{2}\right)$, originating from interacting PDCA units polymers with increasing amount of H-bonding units and different chemical structures. Initially polymers bearing PDCA units on the side chains were tested at a temperature range of -100 to $+100{ }^{\circ} \mathrm{C}$ at a 2 degree/minute scan rate. The $\mathrm{T}_{\mathrm{g}}$ could be extracted either from the peak in $\tan \delta$ or from the loss modulus (E") in DMA: both values are included in Table 3. Two major transitions were observed which can be attributed to the glass temperature $\left(\mathrm{T}_{\mathrm{g}}\right)$ of branched alkyl chains at around $-30{ }^{\circ} \mathrm{C}\left(\mathrm{T}_{\mathrm{g}}^{1}\right)$, and a second transition above $20^{\circ} \mathrm{C}\left(\mathrm{T}_{\mathrm{g}}^{2}\right)$, which is recorded only for polymers having interacting PDCA units and confirmed by AC-chip DSC . Since this transition is also seen in DPP-TVT polymers with PDCA units which have only branched alkyl chains, it can be ruled out that the second transition is representative of the glass transition temperature of linear alkyl chains with bound PDCA units.

By taking the tan delta curves in DMA spectra, we can see that for both classes of polymers with increasing number of $\mathrm{H}$-bonding units present in the alkyl chains and along the backbone, the characteristic peak temperature of the second transition between $20-60{ }^{\circ} \mathrm{C}$ for polymers with PDCA unit raises with increasing H-bonding units contents, as shown in Figure 5a and $5 \mathrm{~b}$ respectively. This is typically attributed to the fact that the film become more crosslinked through intermolecular H-bonding and therefore more thermal energy is required to produce a reduction in film storage modulus. The intensity of the H-bonding transition scales with the amount of mol\% of PDCA in the polymer indicating that the extent of this transition is proportional to the degree of crosslinking of the film which restrict the long range segmental motion of polymer chains. Interestingly, the presence of bonded PDCA units along the alkyl 
chain doesn't have a particular effect on the $T_{g}$ of branched alkyl chains that in all samples tested was found to be constant between $-20{ }^{\circ} \mathrm{C}$ and $-26{ }^{\circ} \mathrm{C}$.

As for both polymers bearing $10 \mathrm{~mol} \%$ of PDCA in the alkyl chains and along the backbone, no notable transition linked to H-bonding breaking has been observed. The transition might therefore overlap with the very broad aliphatic chain $T_{g}$ at lower temperature. In the case of DPP-TVT-10-CB instead, the overall low concentration of bound PDCA units in the already low $10 \mathrm{~mol} \%$ PDCA along the backbone might be also be the reason of the undetectable $\mathrm{H}$ bonding breaking transition. In order to verify the origin of the second transition occurring in polymers bearing PDCA units, we also investigated the thermal behavior of DPP-TVT-60E where similar linear alkyl chains and bulky PDCA-like moieties are introduced. Here, only a very broad transition with a peak at $3{ }^{\circ} \mathrm{C}$ is observed, suggesting that the transitions encountered in the range between $20^{\circ} \mathrm{C}$ and $60^{\circ} \mathrm{C}$ can only be attributed to interacting H-bonding units and not to other events like softening of linear alkyl chains or backbone $\mathrm{T}_{\mathrm{g}}$.

Interestingly, when comparing polymers with the same amount of $\mathrm{H}$-bonding $(30 \mathrm{~mol} \%)$ in the backbone with the same amount in the alkyl chains, the second transition observed was found to be $47^{\circ} \mathrm{C}$ and $26^{\circ} \mathrm{C}$, respectively. Despite the similar chemical structure of the H-bonding units, we found that the location of PDCA units in conjugated polymers greatly affect the amount of bonded vs unbound units and the temperature at which the H-bonding breaking occurs. Indeed, these results suggest that H-bonding, when formed from units present along the rigid backbone, requires more thermal energy to produce a comparable reduction in film storage modulus when compared to the analog unit in alkyl chains. 

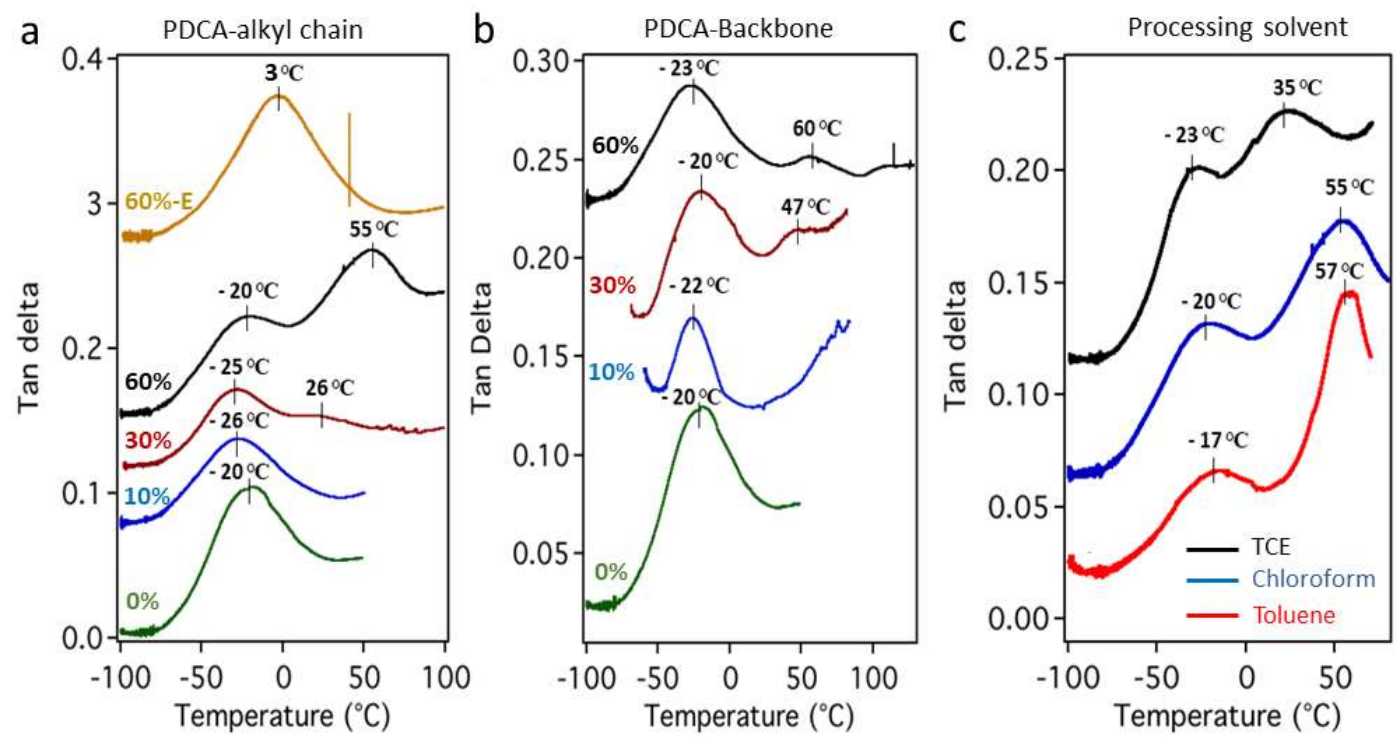

Figure 5. Dynamic mechanical analysis traces (Tan Delta curves) for semiconducting polymers bearing $H$-bonding units in the alkyl chains A) at different wt\% of PDCA (a) DPPTVT-0, b)DPP-TVT-10, c)DPP-TVT-30,d) DPP-TVT-60 and e) DPP-TVT-60E) B) in the backbone (DPP-TVT-0 in green, DPP-TVT-10CB in blue, DPP-TVT-30 red and DPP-TVT-60 in black) and C) $60 \mathrm{wt} \%$ processed from TCE (black curve), chloroform (blue curve) and toluene (red curve). C) Tan delta of DPP-TVT-60 films from different processing solvent ; TCE (black curve), chloroform (blue curve) and toluene (red curve).

Processing conjugated polymers from solvent with different polarity and boiling point was also found to have a profound influence on H-bonding formation and polymorphism. Indeed, it was found that the PDCA units can undergo a cold crystallization process at $\mathrm{T}_{\mathrm{cc}}=50^{\circ} \mathrm{C}$ while melting occurs at $145^{\circ} \mathrm{C}$ (Figure S19). Despite the identical chemical structure of the Hbonding site, the monomer with PDCA inserted along the backbone is amorphous with a clear glass transition temperature at $20^{\circ} \mathrm{C}$. Dynamic mechanical analysis (Figure 5c) revealed that films processed from an apolar solvent like toluene, generate more crystalline $\mathrm{H}$-bonding sites. Instead films processed from polar solvents like chloroform $\left(\mathrm{T}_{\mathrm{b}}=63^{\circ} \mathrm{C}\right)$ and tetrachloroethane $\left(\mathrm{T}_{\mathrm{b}}=154^{\circ} \mathrm{C}\right)$ showed decrease in $\mathrm{H}$-bonding bonding breaking temperature to $50^{\circ} \mathrm{C}$ and $25^{\circ} \mathrm{C}$, 
respectively. Moreover, the stronger interaction occurring between polar solvents and polar group like amides in PDCA can produce a larger distribution in H-bonding conformations during film drying. ${ }^{36}$

Fourier transform infrared spectroscopy. To further investigate the nature of H-bonding among PDCA units in the solid state, attenuated total-reflectance Fourier transformation infrared spectroscopy (ATR-FTIR) was performed. By this method, the presence of H-bonded and un-bound species in the film can be characterized. Thick films were deposited by drop casting of solutions of each polymer from chloroform onto a glass slide and let dry under vacuum overnight. FTIR provides a straightforward and quantitative way to determine the extent of H-bonding in conjugated polymer thin films incorporating H-bonding couples like amine $\left(\sim 3300 \mathrm{~cm}^{-1}\right)$ and carbonyl $\left(\sim 1700 \mathrm{~cm}^{-1}\right)$ functional groups.

The effect of increasing mol\% of PDCA positioned in the alkyl chains and in the conjugated backbone of DPP-TVT on H-bonding formation in thin film was then investigated. Indeed, broad IR peaks in the amide region are attributed to bound protons whereas sharp peaks observed at higher wavenumbers are normally attributed to free NH groups. As shown in Figure 6b, when PDCA units are located on the side chains, almost all the N-Hs are in the H-bonding state $\left(3327 \mathrm{~cm}^{-1}\right)$ already at mol\% as low as $10 \%$. No significant difference is observed in both $\mathrm{C}=\mathrm{O}$ and $\mathrm{NH}$ stretching peak intensity and position for all the polymers with PDCA on the side chains indicating that the H-bonding units are in a bonded state.

In comparison, a high degree of bonding is achieved for polymers with PDCA units along the backbone only at $60 \mathrm{~mol} \%$. Films made of DPP-TVT-10-CB and DPP-TVT-30-CB showed a clearer evolution in intensity and position of the $\mathrm{C}=\mathrm{O}$ stretching with higher amount of PDCA in the structure. At low mol\% of PDCA the $\mathrm{C}=\mathrm{O}$ stretching and $\mathrm{NH}$ stretching consistently 
showed higher intensities for the unbound state peaks at $1740 \mathrm{~cm}^{-1}$ and $3415 \mathrm{~cm}^{-1}$, respectively.
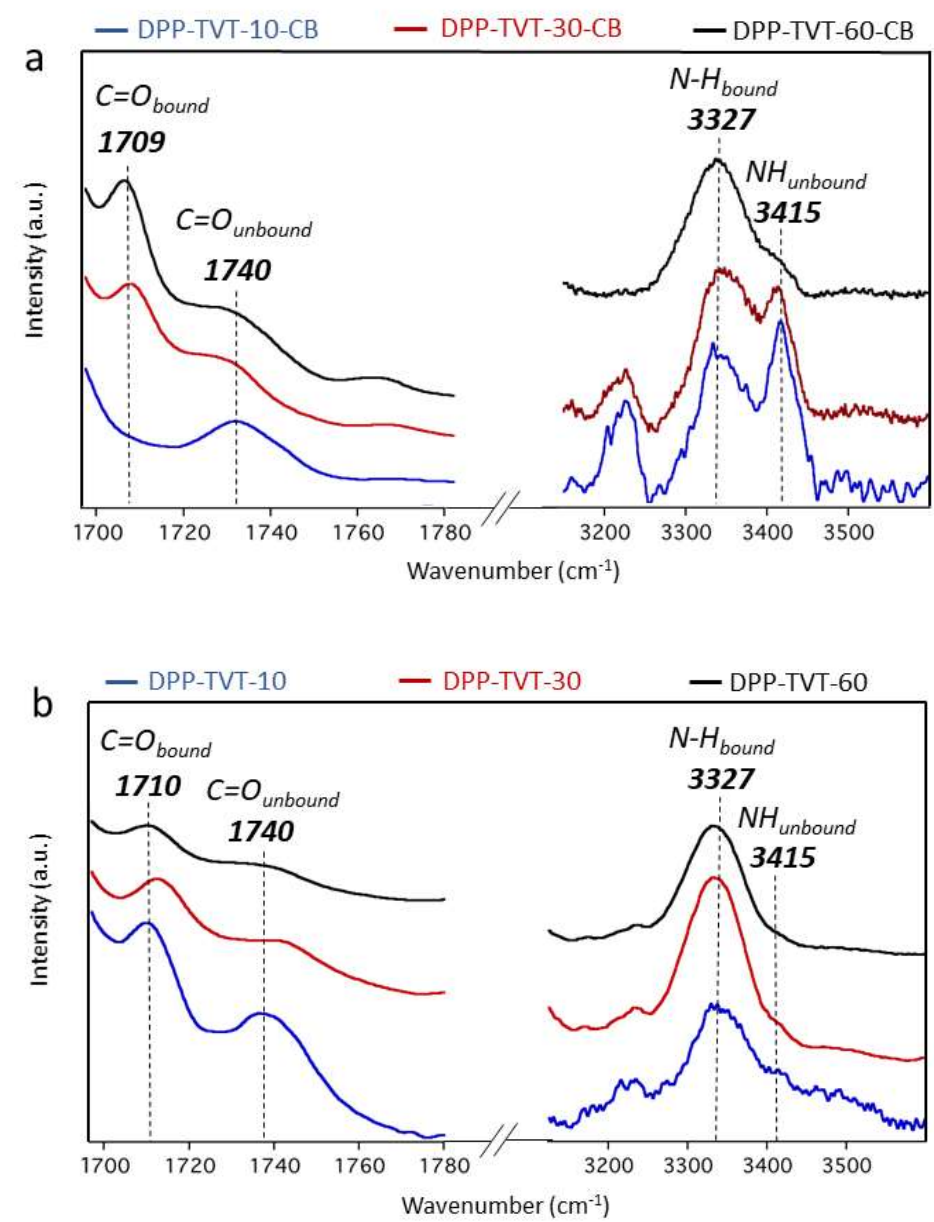

Figure 6. FTIR spectra of solution processed thin films of semiconducting polymers with different H-bonding units (blue line $10 \mathrm{~mol} \%$; red line $30 \mathrm{~mol} \%$ and black $60 \mathrm{~mol} \%$ ) present in the a) backbone and b) in the alkyl chain.

Strain dependent FTIR. For applications in stretchable electronics, semiconducting polymers bearing H-bonding units have been hypothesized to dissipate the energy involved during the stretching by breaking H-bonding units. ${ }^{27}$ In order to verify H-bonding rearrangement, the semiconducting polymer thin films were firstly spin coated onto OTS functionalized silica and transferred onto PDMS substrates. FTIR spectra were subsequently recorded at different 
strains. The ranges of the FTIR spectra that were taken into consideration were the N-H and the $\mathrm{C}=\mathrm{O}$ stretching of the amide functional groups. Semiconducting polymers that showed a thermal transition (DMA) linked to H-bonding rearrangement at RT, such as DPP-TVT-30, were characterized by the appearance of a new and broad shoulder close to the N-H peak and in correspondence with a loosely bound $\mathrm{C}=\mathrm{O}$ stretching at strains higher than the crack onset $(25-30 \%)$ as shown in Figure $7 \mathrm{a}$. The appearance of a broad shoulder in the region of $\mathrm{NH}$ stretching at higher wavenumbers was also found when highly diluted polymers solutions were measured and free NH groups were reported (Figure S27). ${ }^{25}$ DPP-TVT-60, which has a second transition from disruption of hydrogen bonds occurring at temperature higher than RT (peak temperature at $55^{\circ} \mathrm{C}$ ) showed no significant sign of H-bonding breaking or rearrangement even at strains above the crack onset (Figure 7b). Despite the low amount of bounded PDCA, films made of DPP-TVT-10-CB (Figure 7c) showed a small increase in population of un-bound PDCA at increasing strain both from the N-H $\left(3408 \mathrm{~cm}^{-1}\right)$ and $\mathrm{C}=\mathrm{O}\left(1747 \mathrm{~cm}^{-1}\right)$ stretching intensity previously attributed to an unbound state for both functional groups. In earlier section, polymers bearing $30 \mathrm{~mol} \%$ of PDCA units along the backbone, DPP-TVT-30-CB, were found to have the second transition in DMA occurring at a peak temperature greater than room temperature at $47^{\circ} \mathrm{C}$. In this case, thin films of DPP-TVT-30-CB showed no evolution in CO and NH stretching intensity upon strain (Figure 7d), contrary to the behavior reported in DPPTVT-30 films with comparable amount of PDCA in the alkyl chains. Therefore, we can conclude that in order to benefit from energy dissipation from H-bonding re-arrangement, their temperature for H-bonding softening or rearrangement needs to be close to room temperature. But partial breaking or rearrangement during tensile deformation was for the first time proved, by this study, to be possible in conjugated polymers which have a real softening of the crosslinked network already at or near RT as showed for DPP-TVT-30 and DPP-TVT-10-CB. 

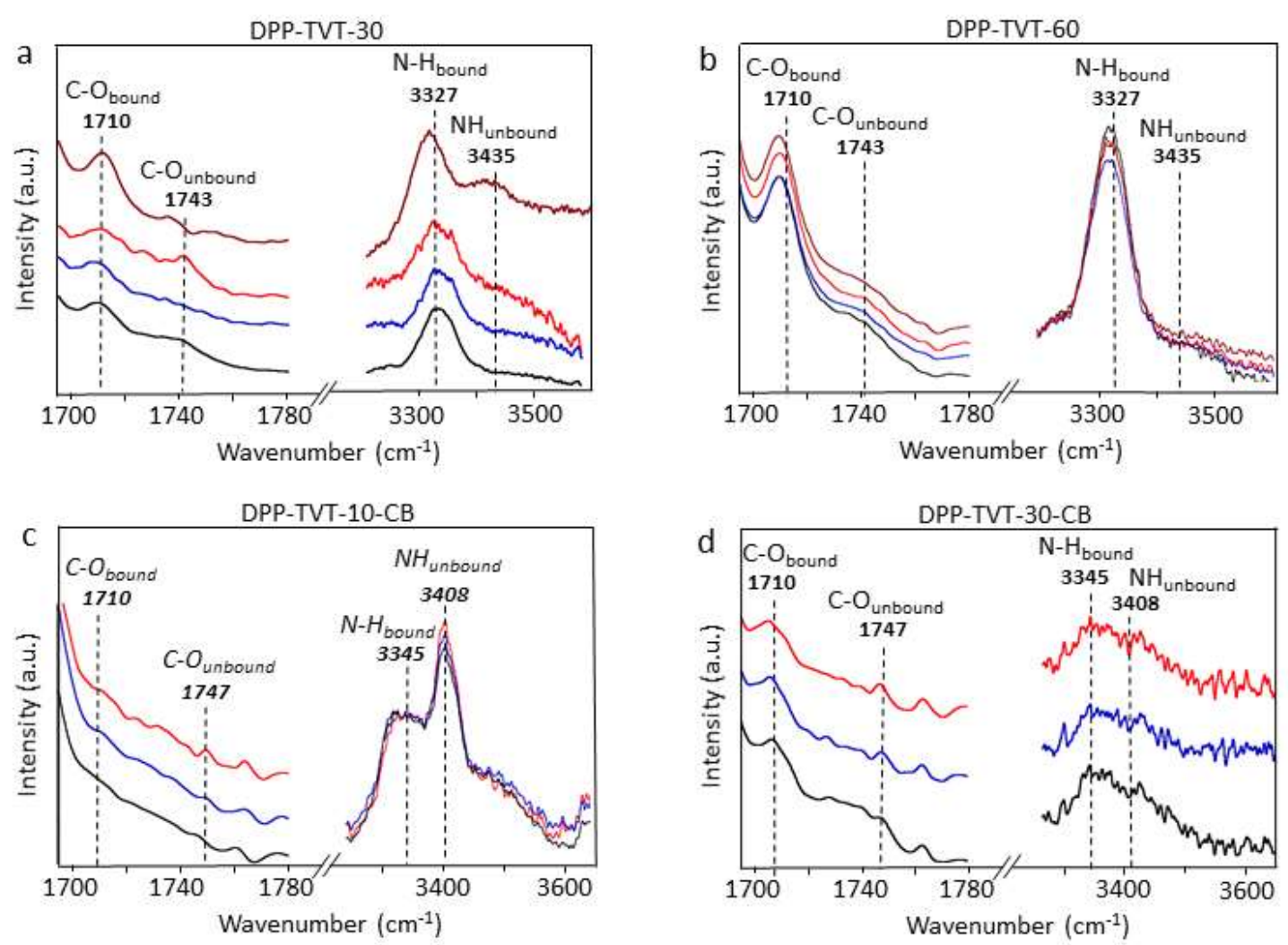

Figure 7. Strain dependent FTIR of DPP-TVT-30 DPP-TVT-60, DPP-TVT-10-CB and DPPTVT-30-CB. Traces in red (50\% strain), in blue (25\% strain) and in black (0\% strain).

\section{Conclusions}

The solubility issue of conjugated polymer bearing PDCA units in side chains encountered by Tsao et al. in previous work was successfully overcome by increasing the linear chain length, which also resulted in improved charge transport and more H-bonding interactions compared to having PDCA units as conjugation breakers in the backbone. The wide range of polymers synthetized with controlled molecular weights and precise amount of PDCA units present in the overall film was important in order to compare thermal and rheological properties between $\mathrm{H}$-bonding polymer with very different chemical structures and degree of cross-linking. From 
FTIR measurements, we were able to determine the degree of bonded and unbonded H-bonding units. Indeed, it was found that DPP polymers bearing PDCA units along the backbone have limited H-bonded units. Fraction of H-bonding was observed only in films of as high as 60 mol\% of PDCA in the backbone. Instead, when the same mol\% of PDCA units are incorporated in the end of linear alkyl side chains, only few PDCA units are unbounded even with $10 \mathrm{~mol} \%$ of PDCA. Increasing the amount of PDCA on the sidechains did not compromise the resulting polymer solubility and charge carrier mobility even though the film was crosslinked through H-bonding.

The location of H-bonding units also has a strong influence on crystalline domain formation. Indeed, we found that when PDCA units are incorporated along the conjugated backbone, bonded units are present mostly in the amorphous regions whereas it is not possible to exclude that free PDCA units can still be present inside crystalline domains as well. Instead, PDCA units in the alkyl chains have a strong influence on crystalline domain formation and amorphous region morphology. Indeed, H-bonded PDCA units can be either found inside crystalline domains (decrease in $\mathrm{T}_{\mathrm{m}}$ of alkyl chains and increase in backbone melting temperature) and in the amorphous regions where increased free volume at room temperature would produce a significant decrease in $\mathrm{T}_{\mathrm{g}}$. From DMA, it was found that for both classes of polymers, an increase in mol\% of PDCA units, hence in cross-linking density, increases the average temperature at which soft cross-linking between H-bonding units are broken. Despite the lower proportion of bound PDCA, DPP-TVT-30-CB showed a H-bonding softening at a much higher temperature $\left(21^{\circ} \mathrm{C}\right.$ higher $)$ compared to DPP-TVT-30 with the same amount of PDCA distributed in the alkyl chains where almost all groups formed H-bonding crosslinking. At higher PDCA loads, the polymer with $60 \mathrm{~mol} \%$ of H-bonding units in the backbone showed a $13^{\circ} \mathrm{C}$ higher transition temperature compared to DPP-TVT-60 analog. 
Processing conditions and in particular solvent polarity and boiling point have an influence on the distribution of possible conformation of H-bonding units. In particular, apolar solvents like toluene, seems to produce films with narrow H-bonding conformations distribution and higher temperature for H-bonding network softening. Under tensile deformation, PDCA units can effectively rearrange as shown from the appearance of weakly bound $\mathrm{C}=\mathrm{O}$ groups, a broadening of the N-H peak and an increase in intensity of the free PDCA N-H stretching peak. However, only polymers with H-bonding softening transition close to RT showed a partial breaking or rearrangement under strain. On the other hand, highly cross-linked films (with high mol $\%$ of PDCA and $\mathrm{T}>50^{\circ} \mathrm{C}$ ) cannot benefit from dissipating energy by deformation or breaking of H-bonded sites. Instead, these systems undergo elastic rearrangements of the amorphous domains prior to crack formation at low strains.

In conclusion, the location and amount of H-bonding units in conjugated polymer can have a significant impact on the thermal and mechanical properties of the resulting polymers. Hbonding breaking was found to occur in DPP-TVT-30 and DPP-TVT-10-CB just prior to crack formation and propagation. The approach presented here can be extended to other polymer backbone structures to gain a more in-depth understanding of the structure-properties relationship of stretchable polymer semiconductors.

\section{References}

1. Webber, M. J., Appel, E. A., Meijer, E. W. \& Langer, R. Supramolecular biomaterials. Nat. Publ. Gr. 15, 13-26 (2016).

2. Grindy, S. C. et al. Control of hierarchical polymer mechanics with bioinspired metalcoordination dynamics. Nat. Mater. 14, 1210 (2015).

3. Wang, S., Oh, J. Y., Xu, J., Tran, H. \& Bao, Z. Skin-Inspired Electronics: An 
Emerging Paradigm. Acc. Chem. Res. 51, 1033-1045 (2018).

4. Rondeau-Gagné, M. U. O. and B. P. C. and A. N. and S. Stretchable electronics: recent progress in the preparation of stretchable and self-healing semiconducting conjugated polymers. Flex. Print. Electron. 2, 43002 (2017).

5. Sun, J. et al. Highly stretchable and tough hydrogels. Nature 489, 133-136 (2012).

6. Sun, T. L. et al. Physical hydrogels composed of polyampholytes demonstrate high toughness and viscoelasticity. Nat. Mater. 12, 932-937 (2013).

7. Barcan, G. a., Zhang, X. \& Waymouth, R. M. Structurally Dynamic Hydrogels Derived from 1,2-Dithiolanes. J. Am. Chem. Soc. 137, 17, 5650-5653 (2015).

8. Guan, X. et al. Development of hydrogels for regenerative engineering. Biotechnol. J. 12, 1600394 (2017).

9. Appel, E. A., del Barrio, J., Loh, X. J. \& Scherman, O. A. Supramolecular polymeric hydrogels. Chem. Soc. Rev. 41, 6195-6214 (2012).

10. Li, C.-H. et al. A highly stretchable autonomous self-healing elastomer. Nat. Chem. 8, 618-624 (2016).

11. Kang, J. et al. Tough and Water-Insensitive Self-Healing Elastomer for Robust Electronic Skin. Adv. Materials 30, 13, 1706846 (2018).

12. Ducrot, E., Chen, Y., Bulters, M., Sijbesma, R. P. \& Creton, C. Toughening Elastomers with Sacrificial Bonds and Watching Them Break. Science 344, 186-189 (2014).

13. Wojtecki, R. J., Meador, M. a \& Rowan, S. J. Using the dynamic bond to access macroscopically responsive structurally dynamic polymers. Nat. Mater. 10, 14-27 (2011).

14. Liu, J. et al. Bioinspired Engineering of Two Di ff erent Types of Sacri ficial Bonds into Chemically Cross-Linked cis -1,4-Polyisoprene toward a High- Performance 
Elastomer. 49, 22, 8593-8604 (2016)

15. Someya, T., Bao, Z. \& Malliaras, G. G. The rise of plastic bioelectronics. Nature 540, 379-385

16. Benight, S. J., Wang, C., Tok, J. B. H. \& Bao, Z. Stretchable and self-healing polymers and devices for electronic skin. Prog. Polym. Sci. 38, 1961-1977 (2013).

17. Lin, Y., Darling, S. B., Nikiforov, M. P., Strzalka, J. \& Verduzco, R. Supramolecular Conjugated Block Copolymers. Macromolecules, 45, 16, 6571-6579 (2012).

18. Lin, Y. et al. Cooperative Assembly of Hydrogen-Bonded Diblock Copolythiophene/Fullerene Blends for Photovoltaic Devices with Well-Defined Morphologies and Enhanced Stability. Chem. Mater. 24, 3, 622-632 (2012).

19. Mei, J. \& Bao, Z. Side Chain Engineering in Solution-Processable Conjugated Polymers. Chem. Mater. 26, 1, 604-615 (2014).

20. Yao, J. et al. Significant Improvement of Semiconducting Performance of the Diketopyrrolopyrrole-Quaterthiophene Conjugated Polymer through Side-Chain Engineering via Hydrogen-Bonding. J. Am. Chem. Soc. 138, 173-185 (2016).

21. Oh, J. Y. et al. Intrinsically stretchable and healable semiconducting polymer for organic transistors. Nature 539, 411 (2016).

22. Tsao, Y. et al. Enhanced Cycling Stability of Sulfur Electrodes through Effective Binding of Pyridine-Functionalized Polymer. ACS Energy Lett. 2, 2454-2462 (2017).

23. Xie, R. et al. Glass Transition Temperature of Conjugated Polymers by Oscillatory Shear Rheometry. Macromolecules 50, 13, 5146-5154 (2017).

24. Inuzuka K., Fujimoto, A. Hydrogen bond energies of 2-aminopyridine dimer and 2aminopyridine-2-pyridone complex formation. Spectrochimica Acta Part A: Molecular Spectroscopy 4, 623-627 (1984).

25. Cellman, S. H., Dado, G. P., Liang, C. \& Adam, B. R. Conformation-Directing Effects 
of a Single Intramolecular Amide-Amide Hydrogen Bond : Variable-Temperature NMR and IR Studies on a Homologous Diamide Series. J. Am. Chem. Soc. , 113, 4, 1164-1173 (1991).

26. Sijbesma, R. P., Genderen, M. H. P. Van, Meijer, E. W. \& February, R. V. Stability and Lifetime of Quadruply Hydrogen Bonded 2-Ureido-4 [ $1 \mathrm{H}$ ] -pyrimidinone Dimers. J. Am. Chem. Soc. 122, 31, 7487-7493 (2000).

27. Oh, J. Y. et al. Intrinsically Stretchable and Healable Semiconducting Polymer for Organic Transistors. Nature 539, 411-415 (2016)

28. Rivnay, J., Noriega, R., Kline, R. J., Salleo, A. \& Toney, M. F. Quantitative analysis of lattice disorder and crystallite size in organic semiconductor thin films. Phys. Rev. $B$ 84, 45203 (2011).

29. Wu, H. C. et al. A rapid and facile soft contact lamination method: Evaluation of polymer semiconductors for stretchable transistors. Chem. Mater. 26, 4544-4551 (2014).

30. Huth, H., Minakov, A. A. \& Schick, C. Differential AC-chip calorimeter for glass transition measurements in ultrathin films. J. Polym. Sci. Part B Polym. Phys. 44, 2996-3005 (2006).

31. Yao, J. et al. Significant Improvement of Semiconducting Performance of the Diketopyrrolopyrrole - Quaterthiophene Conjugated Polymer through Side-Chain Engineering via Hydrogen-Bonding. J. Am. Chem. Soc. 138, 1, 173-185 (2016).

32. He, Y., Zhu, B. \& Inoue, Y. Hydrogen bonds in polymer blends. Prog. Polym. Sci. 29, 1021-1051 (2004).

33. Chen, J. et al. Thickness Dependence of Glass Transitions Measured by AC-Chip Calorimetry in Films with Controlled Interface. Macromolecules 46, 7006-7011 (2013). 
34. Ellison, C. J. \& Torkelson, J. M. The distribution of glass-transition temperatures in nanoscopically confined glass formers. Nat. Mater. 2, 695 (2003).

35. Savagatrup, S., Zhao, X., Chan, E., Mei, J. \& Lipomi, D. J. Effect of Broken Conjugation on the Stretchability of Semiconducting Polymers. Macromol. Rapid Commun. 37, 1623-1628 (2016).

36. Cook, J. L., Hunter, C. A., Low, C. M. R., Perez-velasco, A. \& Vinter, J. G. Solvent Effects on Hydrogen Bonding. Angewandte Chemie International Edition 46, 37063709 (2007).

37. Zhu, B. et al. Thermal and infrared spectroscopic studies on hydrogen-bonding interaction of biodegradable poly(3-hydroxybutyrate)s with natural polyphenol catechin. Green Chem. 5, 580-586 (2003).

38. Rodríguez, M., Molina-Aldareguía, J. M., González, C. \& LLorca, J. Determination of the mechanical properties of amorphous materials through instrumented nanoindentation. Acta Mater. 60, 3953-3964 (2012). 
TOC image

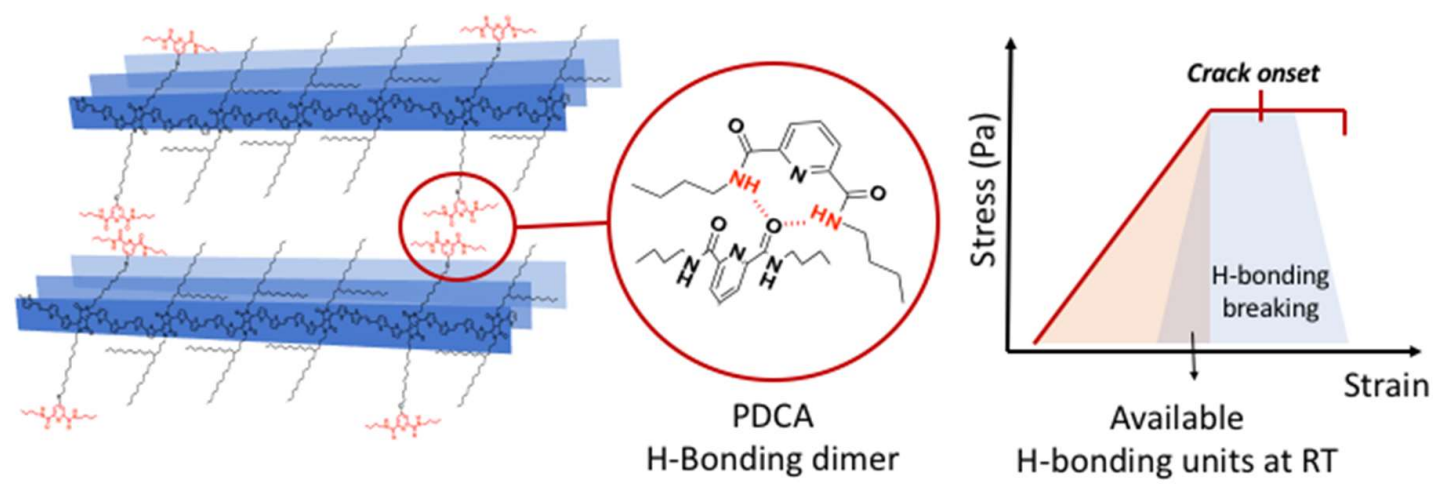

